\title{
A Lightweight Authentication and Key Exchange Protocol for IoT
}

\author{
Abdulrahman BIN Rabiah*, K. K. Ramakrishnan*, Elizabeth Liri* and Koushik Kar ${ }^{\dagger}$ \\ *Department of Computer Science and Engineering, University of California, Riverside \\ Email: abinr001@ucr.edu, kk@cs.ucr.edu, eliri001@ucr.edu \\ †Department of Electrical, Computer and Systems Engineering, Rensselaer Polytechnic Institute \\ Email: koushik@ecse.rpi.edu
}

\begin{abstract}
Security in IoT environments is critical, as there are many situations where IoT devices provide sensory information that needs to be shared securely. However, providing authenticated and secure communication for IoT devices can be a challenge. IoT devices have many constraints, including limitations in computation, power, memory and energy. Moreover, they often have to go through a gateway/sink to connect to the network. For secure communication to the rest of the network, the IoT device needs to trust the gateway/sink, and this requires a means for the device to authenticate the gateway and vice-versa. We also seek to support secure communication even when the IoT device and gateway are disconnected from the rest of the network. In this paper, we provide a lightweight authentication and key exchange protocol for such IoT environments where the IoT device and gateway are communicating over a wireless channel. Our protocol depends on each pair of devices having two unique keys, a master key and an initial session key, provided at configuration time. The session key is constantly changing, and is used as the key for exchanging frames securely during a session. The protocol is lightweight and uses only symmetric-key cryptography and Hashed Message Authentication Code (HMAC)-based key derivation function (HKDF) to provide authentication, key exchange, confidentiality and message integrity. The protocol does not depend on any Trusted Third Party (TTP), and is a good fit for disconnected IoT environments. The keys are never exchanged over the network, providing perfect forward secrecy. The protocol is efficient in the amount of computation required, memory and energy usage.
\end{abstract}

\section{INTRODUCTION}

The use of IoT devices is growing and sensors are becoming increasingly ubiquitous in our lives. There are a number of areas where critical functions, such as monitoring a patient's health status and the environment, depend on data collected from IoT devices. Some functions may frequently send large amounts of data, such as for video surveillance. Such data must be shared in a secure and authenticated manner.

IoT devices are resource constrained, being limited in processing, memory and the availability of power, especially because a lot of them are powered by batteries. They also mostly use wireless as the physical medium of communication. The IoT device uses the gateway/sink as the primary entity

Workshop on Decentralized IoT Security and Standards (DISS) 2018 18 February 2018, San Diego, CA, USA

ISBN 1-891562-51-7

https://dx.doi.org/10.14722/diss.2018.23004

www.ndss-symposium.org through which it communicates with the rest of the environment. For this reason, we seek to have secure and authenticated communication between the IoT device and the gateway/sink that it communicates with. However, traditional authentication and key establishment protocols are not entirely suitable in the IoT environment, because of its specific constraints. Using public key cryptography for authentication and key establishment is not feasible because of the dependence on a Trusted Third Party (TTP), and its high computational requirement. Public key cryptography places a severe demand on compute capability, memory and battery consumption that cannot be met in typical IoT devices. IoT environments may also need to operate in a disconnected mode, with no access to a TTP.

Authentication and key exchange between two nodes without a trusted third party requires some form of a priori establishment of a shared secret between the nodes. In addition, it is important to eliminate the single point of 'failure' (exposure of the secret) in the system. For this reason, we seek to have more than one secret key. Each secret key serves a different purpose. Moreover, we think it is important that the protocol provide Perfect Forward Secrecy (PFS). PFS means that even if the long-term secret key is known somehow at some point, all past session communications must still be secure. In addition, a different session key for each session allows a smaller number of messages encrypted with one session key, making it more difficult for the attacker to find session keys using cryptanalysis. Moreover, even if the attacker is able to find a session key somehow, that session key (being specific to that session) is not useful in decrypting data of future sessions. This motivates the use of limited-life session keys in the lightweight authentication and key exchange protocol for IoT environments that we propose in this paper.

A common practice in IoT security nowadays is to have IoT devices already provisioned with digital certificates, signed by the manufacturer, which are used for authentication and key exchange. However, this common approach requires IoT devices to have high computational capability and high power consumption, as digital certificates rely on public key cryptography. In contrast, we propose using symmetric key cryptography, which is much simpler. Moreover, the common approach requires more memory as public key cryptography uses longer keys compared to symmetric key cryptography and digital certificates, which are a few KB have to be stored. Our proposed protocol has a much smaller memory requirement. Relying on digital certificates without contacting a TTP creates the single point of 'failure' as the only system secret for 
authentication is the private key. If the only system secret is compromised somehow, with this common approach, the authentication and key exchange can be done by the attacker; in contrast, the proposed protocol does not depend solely on one system secret for authentication and key exchange, so even if one of system secrets is found somehow, the authentication and key exchange process cannot be spoofed by the attacker.

The protocol we propose has two keys shared between the IoT device and the gateway: a shared long-term symmetric master key, denoted by $K_{m}$, and a shared short-term symmetric session key, denoted by $K_{s}$. The initial session key, is denoted by $K_{i k s}$. Both the master key and the initial session key are inserted manually once into the IoT device and the gateway/sink. Each gateway authenticates the IoT device and vice versa using messages exchanged between the two devices, which are encrypted using the shared $K_{m}$ and hashed using the shared $K_{i k s}$. Moreover, the protocol ensures that both ends continue to have a shared $K_{s}$, that is used for encrypting and hashing session messages. More importantly, the secret keys are never exchanged explicitly over the network. A re-authentication and update of session key, $K_{s}$, takes place periodically. The messages exchanged between updates is termed a 'session'. Each session has a threshold, which is a maximum number of frames exchanged in that session. The update of the session key, $K_{s}$, is based on a random set of the frames exchanged in the previous session as well as the previous $K_{s}$, and the master key $K_{m}$. As a result, even if the attacker found the $K_{s}$ somehow at some point, he would not be able to calculate next session keys and decrypt messages unless he has both the $K_{m}$ and the current $K_{s}$. Additionally, since it is hard to have a perfect channel where all frames are captured by the attacker [12], our protocol forces the attacker to face three different obstacles. Overcoming the IoT system's security is thus much more difficult.

In addition, our protocol takes into consideration the various constraints and requirements of IoT environments. It uses lightweight mechansims: symmetric key cryptography and a Hashed Message Authentication Code (HMAC)-based Key Derivation Function (HKDF) [2], [6] for confidentiality, message integrity, authentication and key exchange. It seeks to minimize the number of messages (and total bytes exchanged) for authentication and key exchange [3], [7], [9]. It also does not rely on a central, trusted third party in any way. This provides a secure, fast as well as energy and space efficient authentication and key exchange protocol.

The rest of the paper is organized as follows: The next section describes related work. Section III describes the system model and assumptions, including the network attack models considered. Section IV discusses the proposed protocol in detail and Section $\mathrm{V}$ discusses the analysis of the security of the proposed protocol. We conclude in Section VI.

\section{RELATED WORK}

There are a number of authentication and key establishment protocols that have been proposed for use in an IoT environment. Some use public key cryptography for authentication and key establishment [5], [10], which is expensive considering the resource-constraints of IoT devices. Others require authentication and key establishment using a central trusted third party [8], such as a Certificate Authority (CA). However, this is difficult to use in disconnected environments. Another approach is to depend on the hardware capability for introducing randomness for authentication and key establishment using Physically Unclonable Function (PUF) [1]. While the PUF approach is helpful, it is still not yet widely deployed in devices, which prompts us to look at alternatives.

One approach examines key establishment separately [12], utilizing the randomness in wireless channels to update the session key. This approach depends mainly on the reasonable assumption that the wireless channel is not perfect (loss free). The design uses a different session key for each session, a design philosphy that we leverage as well. A session is defined by a threshold number of "One Time Frames" (OTFs), where this number is agreed upon a priori. OTFs are frames transmitted and received exactly once (i.e., without retransmission of those frames). It assumes that both entities start with a publicly well-known fixed value as the initial session key. This key is used to encrypt the session's frames initially. During a session, OTFs are stored at both ends, and after a threshold of OTFs are communicated, the session keys are updated. To update the session key, the current session key and OTFs are used together to incrementally generate a new session key. The design depends on the attacker not having the same channel conditions as the receiver and therefore will see a different subset of OTFs. However, an attacker with a perfect channel (e.g., being very close to the transmitter) makes this scheme susceptible to both passive (e.g., eavesdropping) and active attacks (e.g., hijacking). Our protocol therefore removes this dependency on OTFs and the attacker having an imperfect wireless channel, and builds on the technique proposed in [12].

\section{IOT ENVIRONMENT CONSTRAINTS AND REQUIREMENTS}

IoT environments are associated with a number of constraints. Foremost, IoT devices have limited energy as they are primarily dependent on batteries (e.g., disposable coin cell batteries that might last for 1-2 years at most with limited use). Second, IoT devices have limited processing capabilities and limited memory, with only a few KB of RAM and tens of $\mathrm{KB}$ of EEPROM. IoT devices rarely have fast re-writable non-volatile data storage.

In addition, the IoT device and gateway may have to operate in a disconnected environment, limiting access to a central entity or a trusted third party. Finally, the approach for managing these devices needs to be scalable as there are likely to be a very large number of IoT devices, and it is important to have as little human intervention as possible.

\section{A. Network Model and Assumptions}

The generic network topology that we consider is shown in Figure 1. There are multiple IoT devices and a single Gateway, and each IoT device communicates with other nodes only through the Gateway. We assume, in this paper, that MAC layer protocol on the wireless link delivers packets reliably, in-sequence.

A new IoT device added to this IoT network will first have to authenticate with the gateway and vice-versa to establish communication with the rest of the environment. We assume 


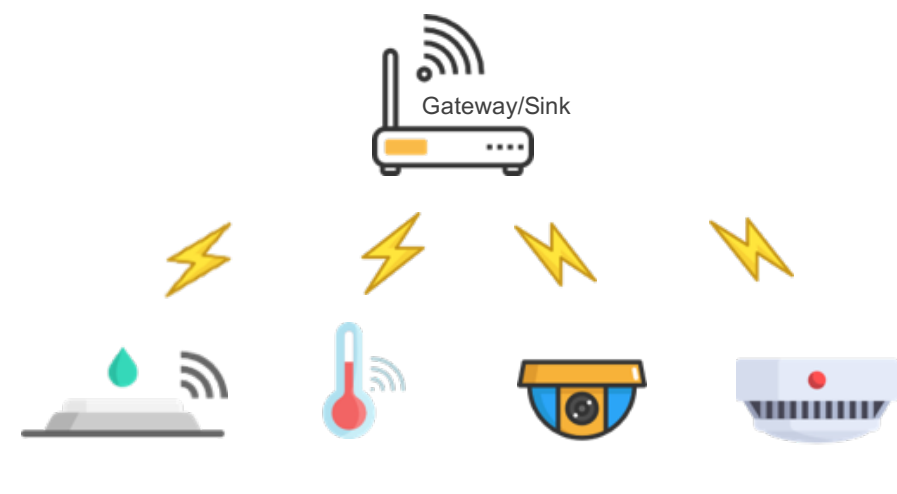

Fig. 1: Network Topology

that both the IoT device and the gateway have a limited amount of non-volatile storage in the form of an EEPROM that can store shared keys. We further assume that a limited amount of manual configuration is feasible, so that the administrator can set up shared keys for the gateway and IoT device on a pairwise basis. We assume that an attacker does not have physical access to the devices and thus cannot access the configured keys that are stored on the devices.

\section{B. Attack Scenarios}

In this section, we consider the range of possible ways that an attacker with access to the link may seek to exploit vulnerabilities in the protocol we design. We then describe (briefly) how these attacks are prevented by our protocol described in this paper.

1) An attacker may try to sniff or modify session messages.

2) An attacker may seek to discover the secret keys by snooping on the authentication and key exchange traffic.

3) An attacker may try to modify or spoof authentication and key exchange messages to cause disruptions.

4) An attacker may try to launch replay attacks to cause disruptions.

5) An attacker may launch man-in-the-middle attacks and have unauthorized access to confidential messages and possibly alter them.

We believe that our protocol is designed to thwart all of these attacks, and we have performed a security analysis of the protocol (described briefly in Section V) to verify this.

\section{Lightweight Authentication And Key EXCHANGE PROTOCOL}

As mentioned earlier, for each Gateway-IoT device pair, we maintain two shared secret keys: a shared long-term symmetric master key, called $K_{m}$; and a second, shared short-term symmetric session key, called $K_{s}$. Initially, the $K_{m}$ and $K_{s}$ are manually inserted into both devices, in non-volatile storage such as EEPROM. The specific initial value of $K_{s}$, that is added manually is denoted by $K_{i k s}$. Each IoT device and Gateway pair has a unique pair of $K_{m}$ and $K_{i k s}$, specific for their use and both $K_{m}$ as well as $K_{i k s}$ play an important role in authentication in our protocol. IoT devices and the Gateway are authenticated using messages encrypted with the shared $K_{m}$ and hashed with the shared $K_{i k s}$ while both nodes use the shared $K_{s}$ to encrypt and hash messages during a session. The protocol makes use of authenticated encryption, namely the Counter with CBC-MAC (CCM) block cipher mode [4], [11], to ensure confidentiality and message authentication in all phases. With CCM mode, CBC-MAC is used to calculate a Message Authentication Code (MAC) for the whole frame (header, nonce and payload) using a symmetric secret key (either $K_{m}$ or $K_{s}$, depending on the phase) and the Counter mode is used to encrypt the payload as well as the MAC using a nonce and symmetric secret key (either $K_{i k s}$ or $K_{s}$, depending on the phase) whereas the header needs to be in plaintext so that the other end can learn some information about the frame in order to process it, such as the MAC addresses. A new session begins when the number of session frames exchanged during the previous session reaches a preset threshold (agreed upon a priori by both sides).

$K_{s}$ is updated regularly for each session, and is never exchanged explicitly over the network. In addition when updating $K_{s}$, the previous value of $K_{s}$ is used, so that the value of $K_{s}$ is cumulative, meaning the new session key $K_{s}$ depends on the previous session's $K_{s}$.

The protocol we propose takes advantage of the randomness in regular frames and uses it to generate more random session keys. Both entities integrate randomness by incorporating a randomly selected set of future session frames into a newly generated $K_{s}$. This set of future session frames is agreed on and communicated securely using another secret key, $K_{m}$, every time a $K_{s}$ update takes place. Since the frame threshold is known by both entities, the range of frame sequence numbers till the next $K_{s}$ update can be calculated. Using a random number generator, the Gateway selects a random set of future frame sequence numbers within this range so that both entities keep only those frames in a buffer. For each session, the larger the number of frame sequence numbers selected, the more random the new $K_{s}$ is. This randomness provides better security for the exchange of $K_{s}$. However, we note that IoT devices have limited memory, which can limit the number of session frames they can buffer.

Thus, with every new session, it becomes harder for the attacker to keep up with the $K_{s}$ updates. To violate confidentiality, the attacker would need to have both the $K_{m}$ and $K_{s}$. Although it is hard to have a perfect channel where all frames are captured [12], we make no assumption about the ability of the attacker to have or not have a perfect channel to capture all the frames sent by the IoT device or the Gateway. When there is a failure in synchronization of the $K_{s}$ at either end for any reason, whether malicious or non-malicious, either device can always initiate a reset of the $K_{s}$ to a new random key derived from $K_{i k s}$, along with nonces, so they can always have a stable communication channel.

\section{A. Initial Setup Phase for a New IoT Device}

When an IoT device first joins the network, the initial setup needed for both the IoT device and the gateway is to authenticate each other and negotiate a $K_{s}$. This is done using the shared $K_{m}$ and the $K_{i k s}$ that have been installed manually 


\begin{tabular}{|c|c|}
\hline Acronym & Definition \\
\hline$K_{m}$ & Shared long-term symmetric master key \\
\hline$K_{s}$ & Shared short-term symmetric session key \\
\hline$K_{i k s}$ & Initial $K_{s}$ \\
\hline$I D_{I}$ & IoT device ID \\
\hline$I D_{G}$ & Gateway ID \\
\hline Nonce $_{1}$ & Randomly generated value used once \\
\hline Nonce $_{2}$ & Randomly generated value used once \\
\hline RandFrmSeqs ${ }_{i}$ & Random set of sequence numbers of session $(i-1)$ frames to be used in session $i$ 's session key update \\
\hline RandFrmSeqs $s_{i+1}$ & Random set of sequence numbers of session $i$ frames to be used in session $(i+1)$ 's session key update \\
\hline $\operatorname{RandFrm}_{i}$ & Random frames from last session (session $(i-1)$ ) based on $\operatorname{RandFrmSeqs}_{i}$ \\
\hline KeyLength & Desired length of the derived key \\
\hline
\end{tabular}

TABLE I: Glossary

into both the gateway and new IoT device. Figure 2 shows the protocol interaction.

\begin{tabular}{|c|c|c|}
\hline \multicolumn{2}{|c|}{ IoT Device } & Gateway \\
\hline \multirow{4}{*}{$\begin{array}{l}\text { loT device } \\
\text { authenticates } \\
\text { Gateway. }\end{array}$} & $\begin{array}{l}\text { ID }_{1} \text {, 'initialization', } E_{K_{m}} \text { (Nonce1), } \\
E_{K_{m}}\left(M A C_{K_{i k s}}(\text { Message } 1)\right)\end{array}$ & \multirow{3}{*}{$\begin{array}{l}\text { Message } 1 \\
\text { Message } 2\end{array}$} \\
\hline & $\begin{array}{l}\mathrm{ID}_{\mathrm{G}}, E_{K_{m}}(\text { Nonce2, } \\
\left.\text { RandFrmSeqs }_{i+1}, \text { Nonce } 1\right), \\
E_{K_{m}}\left(M_{A} C_{K_{i k s}} \text { (Message 2)) }\right.\end{array}$ & \\
\hline & $\begin{array}{l}\text { iD }, E_{K_{m}}(\mathrm{~h}(\text { Nonce2 } \| \\
\left.\left.\text { RandFrmSeqs } \mathrm{s}_{\mathrm{i}+1}\right)\right), \\
E_{K_{m}}\left(M_{A} C_{K_{i k s}}(\text { Message 3)) }\right.\end{array}$ & \\
\hline & $\begin{array}{l}\mathrm{ID}_{\mathrm{G}}, E_{K_{m}} \text { ('Ack'), } \\
E_{K_{m}}\left(M A C_{K_{i k s}}(\text { Message 4)) }\right.\end{array}$ & $\begin{array}{l}\text { Gateway } \\
\text { authenticates loT } \\
\text { device. } \\
\text { Message } 4\end{array}$ \\
\hline $\begin{array}{l}K_{S}=\mathrm{HKDF}_{K_{\text {dks }}} \text { (Nonce1 } \\
\| \text { Nonce2, } \\
\text { KeyLength). } \\
\text { Session message } \\
\text { exchanges start }\end{array}$ & & $\begin{array}{l}K_{S}=\mathrm{HKDF}_{K_{i k s}} \text { (Nonce1 ॥ } \\
\text { Nonce2, KeyLength). } \\
\text { Session message } \\
\text { exchanges start }\end{array}$ \\
\hline
\end{tabular}

Fig. 2: Initial Setup Phase

a) Message 1: The IoT device sends its $I D_{I}$, an 'initialization', a Nonce1 and $\mathbf{M A C}_{K_{i k s}}$ (Message 1), encrypted with the $K_{m}$ to the gateway. In order to prove its identity, the authenticator, which is the IoT device at this point, provides a new challenge, Nonce1, that will be used by the supplicant, Gateway. Additionally, using $K_{i k s}$ to calculate the MAC for the whole message, including the nonce verifies both the data integrity and the authenticity of the message, thus helping to prevent disruptions that may be caused by an attacker who has determined the $K_{m}$ somehow.

b) Message 2: Upon receipt of the first message from the IoT device, the Gateway first selects a random set of sequence numbers of future frames, RandFrmSeqs $s_{i+1}$, to be considered when updating the $K_{s}$ next time. This is communicated, along with a Nonce2 (a new challenge picked by the Gateway) and Nonce1, to the IoT device, as part of Message 2. The Nonce2, RandFrmSeqs $s_{i+1}$ and Nonce1 are encrypted with the master key, so that it is confidentially exchanged with the IoT device. Thus, when this session starts, the IoT device will retain a copy of frames with those sequence numbers to be used in the next $K_{s}$ update.

In order for the IoT device to authenticate the Gateway and also confirm that it received the right set of
RandFrmSeqs $_{i+1}$, the Gateway also calculates the MAC of the whole message, including Nonce1 and RandFrmSeqs $s_{i+1}$ using the $K_{i k s}$ as a key and sends it to the IoT device. The IoT device can thus verify that the Gateway has the correct $K_{i k s}$ and has received Noncel correctly, when it calculates the corresponding MAC using $K_{i k s}$. All the parameters sent by the Gateway, $I D_{G}$, the encrypted Nonce2, along with the RandFrmSeqs $s_{i+1}$, and the Nonce1 are encrypted using $K_{m}$ and hashed using $K_{i k s}$.

c) Message 3: When the IoT device receives Message 2 , it verifies that the hash value of the MAC, that it just received matches what it calculates based on the decrypted message using $K_{m}$. The IoT device marks the Gateway as authenticated and confirms that the IoT device has received the right set of RandFrmSeqs $s_{i+1}$. Moreover, the IoT device verifies that the Gateway has the correct $K_{i k s}$ and has received Nonce1 correctly from Message 1, thus authenticating the Gateway.

The IoT device calculates the hash of Nonce2 and RandFrmSeqs $_{i+1}$. $\mathbf{h}$ (Nonce2||RandFrmSeqs $s_{i+1}$ ) and $\mathbf{M A C}_{K_{i k s}}$ (Message 3) are communicated to the Gateway for it to authenticate the IoT device and for it to confirm that the RandFrmSeqs $s_{i+1}$ was received correctly by the IoT device. The IoT device sends $I D_{I}$, and the encryption of $\mathbf{h}\left(\right.$ Nonce2 $_{\mid}$RandFrmSeqs $_{i+1}$ ) and $\mathbf{M A C}_{K_{i k s}}$ (Message 3) using $K_{m}$.

d) Message 4: When the Gateway receives Message 3 , it verifies the MAC. If valid, the Gateway marks the IoT device as authenticated, and it knows the IoT device has received the right set of RandFrmSeqs $s_{i+1}$. This also lets the Gateway know that the IoT device has the correct $K_{i k s}$ and has received Nonce 2 correctly from Message 2 . The Gateway sends $I D_{G}$, the encryption of an ack and $\mathbf{M A C} \mathbf{K}_{i k s}$ (Message 4) using $K_{m}$ to the IoT device for it to confirm that Message 3 was received correctly by the Gateway. The Gateway now sets $K_{s}$ to a random key derived from Nonce1 and Nonce2, used as a salt input (a random value), using $K_{i k s}$ as a HKDF key.

When the IoT device receives Message 4, it verifies the MAC. If valid, the IoT device knows Message 3 was received correctly. The IoT device sets $K_{s}$ to the random key derived from Nonce1 and Nonce2 using $K_{i k s}$ as a HKDF key. 


\section{B. Normal Communication between Gateway and IoT Device}

During normal communication between the IoT device and Gateway, both devices have agreed upon a $K_{s}$, and they exchange messages securely using $K_{s}$. During this phase, both devices keep the frames corresponding to the current RandFrmSeqs $s_{i+1}$ to be used for the next $K_{s}$ update until the threshold of frames exchanged is reached. Not only is the RandFrmSeqs $s_{i+1}$ not known to the adversary, it is also possible that not all of the frames of the session received by the adversary. IoT link layer protocols such as IEEE 802.15.4 are also capable of protecting the confidentiality and integrity of data. Therefore, our protocol provides the link layer with the $K_{s}$ for this purpose. An approximate session interaction is shown in Figure 3. Normal session frames are encrypted and hashed with the $K_{s}$ and sent to the other end. An ack, encrypted and hashed with the $K_{s}$, is sent by the receiver to acknowledge correct receipt of a frame. When the threshold of frames exchanged is reached, we go to the next phase for update of $K_{s}$.

\begin{tabular}{|c|c|c|}
\hline \multicolumn{2}{|c|}{ IoT Device } & \multirow{4}{*}{$\begin{array}{l}\text { Message i } \\
\text { Gateway sends } \\
\text { acknowledgments } \\
\text { Ack i }\end{array}$} \\
\hline \multirow[t]{5}{*}{$\begin{array}{l}\text { loT device sends } \\
\text { session data }\end{array}$} & $E_{K_{s}}($ Data $), E_{K_{s}}\left(M A C_{K_{s}}(\right.$ Message i $\left.)\right)$ & \\
\hline & $E_{K_{s}}($ Ack $), E_{K_{s}}\left(M A C_{K_{s}}(\right.$ Ack i $\left.)\right)$ & \\
\hline & $\begin{array}{c}\vdots \\
E_{K_{s}}(\text { Data }), E_{K_{s}}\left(M A C_{K_{s}}(\text { Message } \mathrm{n})\right)\end{array}$ & \\
\hline & $E_{K_{s}}(\mathrm{Ack}), E_{K_{s}}\left(M A C_{K_{s}}(\right.$ Ack n $\left.)\right)$ & \multirow{2}{*}{$\begin{array}{l}\text { Message } n \\
\text { Ack } n\end{array}$} \\
\hline & & \\
\hline
\end{tabular}

Fig. 3: Normal Communication between Gateway and IoT Device

\section{Session Key Update}

The update of $K_{s}$ is shown in Figure 4.

\begin{tabular}{|c|c|c|}
\hline \multicolumn{2}{|c|}{ IoT Device } & ateway \\
\hline $\begin{array}{l}K_{s}=\operatorname{HKDF}_{K_{s}}\left(K_{m}\right. \\
\text { RandFrmm } \\
\text { KeyLength) }\end{array}$ & $\begin{array}{l}\text { ID }, \text { 'update key', } \\
E_{K_{m}}\left(\text { Nonce1), } E_{K_{m}}\left(M A C_{K_{s}} \text { (Message1)) }\right.\right.\end{array}$ & Message 1 \\
\hline & $\begin{array}{l}\text { ID }_{G}, E_{K_{m}}(\text { Nonce2, RandFrmSeqs } \\
\text { Nonce1 } 1), E_{K_{m}}\left(M A C_{K_{s}} \text { (Message 2)) }\right.\end{array}$ & $\begin{array}{l}K_{s}=\mathrm{HKDF}_{K_{s}}\left(K_{m},\right. \\
\text { RandFrm }, \\
\text { KeyLength })\end{array}$ \\
\hline $\begin{array}{l}\text { loT device } \\
\text { authenticates } \\
\text { Gateway }\end{array}$ & 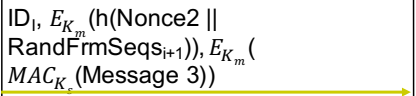 & \\
\hline & & $\begin{array}{l}\text { Gateway } \\
\text { authenticates loT } \\
\text { device }\end{array}$ \\
\hline $\begin{array}{l}\text { Session message } \\
\text { exchanges start }\end{array}$ & & $\begin{array}{l}\text { Session message } \\
\text { exchanges start }\end{array}$ \\
\hline
\end{tabular}

Fig. 4: Session Key Update

a) Message 1: The IoT device calculates $K_{s}$ for the next session, which will be the HKDF of random frames based on RandFrmSeqs $s_{i}$, used as an information input (context and application specific information), that it received in the last session, and $K_{m}$, used as a salt input, using the current $K_{s}$. Then, the IoT device sends $I D_{I}$, the command to 'update key' and Nonce1, along with $\mathbf{M A C}_{K_{s}}$ (Message 1) encrypted with the $K_{m}$ to the Gateway.

b) Message 2: When the Gateway receives the update key message, it verifies the threshold has been reached as well as the MAC. It then calculates $K_{s}$ for the new session, as the HKDF of the random frames based on RandFrmSeqs $i$, used as an information input, and $K_{m}$, used as a salt input, using the current $K_{s}$. It calculates the MAC using $K_{s}$ as a key which is communicated to the IoT device in this step. The Gateway sends $I D_{G}$ and the encryption of Nonce2, RandFrmSeqs $s_{i+1}$, Nonce1 and $\mathbf{M A C}_{K_{s}}$ (Message 2) using the $K_{m}$ to the IoT device.

c) Message 3: When the IoT device receives Message 2, it marks the Gateway as authenticated by verifying the MAC as in Sec IV-A. It calculates the hash of Nonce 2 and RandFrmSeqs $s_{i+1}$ to match the action at the Gateway. The IoT device sends $I D_{I}$ and the encryption of $\mathbf{h}$ (Nonce2||RandFrmSeqs $s_{i+1}$ ) and $\mathbf{M A C}_{K_{s}}$ (Message 3) using $K_{m}$. When the Gateway receives Message 3, it marks the IoT device as authenticated once the MAC is verified, and this completes the update phase.

\section{Mutual Re-Authentication and Re-Establishment of Session Keys}

When there is a failure in synchronization of $K_{s}$ at either end for any reason, whether malicious or otherwise, both devices can renegotiate $K_{s}$ securely. Recall that both $K_{m}$ and the $K_{i k s}$ are maintained in both devices in non-volatile memory throughout. Thus, both devices use $K_{m}$ and $K_{i k s}$ to re-authenticate and re-establish a newly generated random $K_{s}$ derived from Nonce1, Nonce2 and $K_{i k s}$, which makes $K_{s}$ to be different with high probability, each time a reset of $K_{s}$ is performed. For example, the IoT device or Gateway may reboot for any reason, resulting in both ends having to reset their $K_{s}$ to a new random $K_{s}$ and re-authenticate and re-establish the session key securely, as shown in Figure 5.

\begin{tabular}{|c|c|c|}
\hline \multicolumn{2}{|c|}{ loT Device } & Gateway \\
\hline & $\begin{array}{l}\text { ID }_{1} \text {,'reset', } E_{K_{m}}(\text { Nonce } 1), \\
E_{K_{m}}\left(M A C_{K_{i k s}}(\text { Message 1) })\right.\end{array}$ & \multirow{3}{*}{ Message 2} \\
\hline \multirow{3}{*}{$\begin{array}{l}\text { loT device } \\
\text { authenticates } \\
\text { Gateway. }\end{array}$} & $\begin{array}{l}\mathrm{ID}_{\mathrm{G}}, E_{K_{m}}(\text { Nonce2 } 2, \\
\left.\operatorname{RandFrmSeqs}_{i+1}, \text { Nonce } 1\right) \text {, } \\
E_{K_{m}}\left(M A C_{K_{i k s}}(\text { Message } 2)\right)\end{array}$ & \\
\hline & $\begin{array}{l}\text { ID }, E_{K_{n}}(\mathrm{~h}(\text { Nonce2 } \| \\
\text { RandFrmSeqs } \\
E_{K_{m}}\left(M A C_{K_{i k}}\left(M_{1}\right)\right) \\
\text { (Message 3)) }\end{array}$ & \\
\hline & $\begin{array}{l}\mathrm{ID}_{\mathrm{G}}, E_{K_{m}}(\text { 'Ack') }, \\
E_{K_{m}}\left(M A C_{K_{i k s}}(\text { Message 4)) }\right.\end{array}$ & \multirow{2}{*}{$\begin{array}{l}\text { Message } 3 \\
\text { Gateway } \\
\text { authenticates loT } \\
\text { device. } \\
\text { Message } 4 \\
K_{s}=\text { HKDF }_{K_{u s}} \text { (Nonce1 II } \\
\text { Nonce2, KeyLength). }\end{array}$} \\
\hline $\begin{array}{l}K_{s}=\mathrm{HKDF}_{K_{i k s}} \text { (Nonce1 } \\
\| \text { Nonce2, } \\
\text { KeyLength). }\end{array}$ & & \\
\hline $\begin{array}{l}\text { Session message } \\
\text { exchanges start }\end{array}$ & & $\begin{array}{l}\text { Session message } \\
\text { exchanges start }\end{array}$ \\
\hline
\end{tabular}

Fig. 5: Mutual Re-Authentication and Re-Establishment of Session Keys

This process is very similar to the initial authentication and key establishment discussed in Section IV-A, except for 
the command to 'reset' instead of 'initialization'. The main distinction is that all outstanding frames are flushed from the memory at both devices so that both devices have same frames for the next update of $K_{s}$.

\section{SECURITy ANALYSis}

We have done a careful security analysis of the proposed protocol for a range of possible attacks that was described in Section III-B. We believe that having both the keys, $K_{m}$ and $K_{s}$ provide better security as it makes it more difficult for the attacker to derive both keys. Our analysis showed that knowing just one of the two keys is not sufficient to violate the confidentiality and message integrity of our protocol. We also were able to show that the proposed protocol satisfies the important perfect forward secrecy (PFS) property. We highlight a few key points here:

1) The length of $K_{m}$ and $K_{s}$ makes it difficult to use brute force tactics to find them.

2) Knowing $K_{m}$ alone is not sufficient to decrypt session messages since they are encrypted using another key.

3) The session keys are never explicitly exchanged in the messages; knowing $K_{m}$ is not enough to know $K_{s}$.

4) Even if an attacker knows $K_{m}$, has a perfect channel, and has recorded all messages exchanged from the very beginning, they still cannot obtain a $K_{s}$ because the keys are never exchanged explicitly over the network. In addition, the attacker cannot calculate current $K_{s}$ because it depends on the previous $K_{s}$.

5) If an attacker knows $K_{s}$ and even if he has a perfect channel, he cannot know the agreed upon random frames that will be used to construct the next session's key.

6) If only $K_{m}$ is known and the IoT device is rebooted or the channel is jammed by the attacker who also records all the messages exchanged, the protocol remains robust against man-in-the-middle attacks and session hijacking.

7) If the attacker only knows $K_{m}$ at the initial setup phase, man-in-the-middle or session hijack attacks still cannot succeed because the attacker has to have the other key, $K_{i k s}$, to prove it is the legitimate entity to the other end.

We do not include the details of our security analysis due to space limitations.

\section{CONCLUSIONS}

We propose a lightweight authentication and key exchange protocol to help secure communication in IoT environments that typically communicate over wireless channels. The proposed protocol aims to provide secure, lightweight, energy and space efficient authentication and key exchange for resourceconstrained devices without depending on a central trusted third party. The protocol's main strengths include having secret keys that are not explicitly exchanged over the air. The protocol frequently updates the symmetric session key used for encrypting and hashing the data exchanged based on a random (but previously agreed upon) number of frames of a session. We also introduce two obstacles that have to be compromised to break the protocol, namely a symmetric long-term key $\left(K_{m}\right)$, a symmetric short-term key $\left(K_{s}\right)$. In the proposed protocol, therefore, there is no single component that can be exploited by an attacker to compromise the authentication and key exchange protocol or the confidentiality and integrity of the information exchanged. For future work, we will enhance our protocol to require only one pre-shared key and still achieve PFS, but it would need to rely on a commit protocol. We are currently working on accommodating lossy links.

\section{ACKNOWLEDGMENT}

This work was supported in part by NSF grant CNS1619441 and a gift from Futurewei Technologies.

\section{REFERENCES}

[1] M. N. Aman, K. C. Chua, and B. Sikdar, "Secure data provenance for the internet of things," in Proceedings of the 3rd International Workshop on IoT Privacy, Trust, and Security, ser. IoTPTS '17. ACM, 2017, pp. 11-14.

[2] M. Bellare, R. Canetti, and H. Krawczyk, "Keying hash functions for message authentication," in Crypto, vol. 96. Springer, 1996, pp. 1-15.

[3] L. Casado and P. Tsigas, "Contikisec: A secure network layer for wireless sensor networks under the contiki operating system," Identity and Privacy in the Internet Age, pp. 133-147, 2009.

[4] P.-A. Fouque, G. Martinet, F. Valette, and S. Zimmer, "On the security of the ccm encryption mode and of a slight variant," in Applied Cryptography and Network Security. Springer, 2008, pp. 411-428.

[5] H. R. Hussen, G. A. Tizazu, M. Ting, T. Lee, Y. Choi, and K.-H. Kim, "Sakes: Secure authentication and key establishment scheme for $\mathrm{m} 2 \mathrm{~m}$ communication in the ip-based wireless sensor network (610wpan)," in Proceedings of Fifth International Conference on Ubiquitous and Future Networks (ICUFN), July 2013, pp. 246-251.

[6] H. Krawczyk, "Cryptographic extraction and key derivation: The hkdf scheme." in CRYPTO, vol. 6223. Springer, 2010, pp. 631-648.

[7] P. Mahajan and A. Sachdeva, "A study of encryption algorithms aes, des and rsa for security," Global Journal of Computer Science and Technology, 2013.

[8] P. Porambage, C. Schmitt, P. Kumar, A. Gurtov, and M. Ylianttila, "Pauthkey: A pervasive authentication protocol and key establishment scheme for wireless sensor networks in distributed iot applications," International Journal of Distributed Sensor Networks, vol. 10, no. 7, p. 357430, 2014.

[9] N. R. Potlapally, S. Ravi, A. Raghunathan, and N. K. Jha, "Analyzing the energy consumption of security protocols," in Proceedings of International Symposium on Low Power Electronics and Design, ser. ISLPED '03. ACM, 2003, pp. 30-35.

[10] Y. Qiu and M. Ma, "A mutual authentication and key establishment scheme for $\mathrm{m} 2 \mathrm{~m}$ communication in 6lowpan networks," IEEE Transactions on Industrial Informatics, vol. 12, no. 6, pp. 2074-2085, Dec 2016.

[11] D. Whiting, R. Housley, and N. Ferguson, "Counter with cbcmac (ccm)," Internet Requests for Comments, RFC Editor, RFC 3610, September 2003. [Online]. Available: http://www.rfceditor.org/rfc/rfc3610.txt

[12] S. Xiao, W. Gong, and D. Towsley, "Secure wireless communication with dynamic secrets," in Proceedings of IEEE INFOCOM 2010, March 2010, pp. 1-9. 